\title{
Features of Sensorineural Hearing Loss Caused by Exposure to Aircraft Noise
}

\author{
PM Sheshegov ${ }^{1 *}$ and VN Zinkin ${ }^{2}$ \\ ${ }^{1}$ doctor honey sciences, Russia \\ ${ }^{2}$ doctor med of sciences, Russia
}

*Corresponding author: PM Sheshegov, doctor honey sciences, Russia.

Received Date: June 26, 2020

Published Date: July 08, 2020

\section{Short Communication}

An urgent problem in industry and transport continues to be the adverse effects of noise. In terms of prevalence among industrial physical factors, noise in Russia ranks first (17.5\%). Exposure to noise with a level exceeding the maximum permissible level, several million people are exposed. Among occupational diseases from physical factors, sensorineural hearing loss (STI) steadily takes the first place, and its share in 2018 was 55\%. When studying the working conditions of aviation specialists (AS), the spectrum of aviation noise (AS) contains an infrasound component, the role of which was not given much importance in the formation of aviation pathology. It is shown that the simultaneous action of noise and infrasound (FR) exacerbates the harmful effects of each of these factors. Despite numerous studies on the adverse effects of AS, the mechanisms of its action on humans require further study.

The main source of noise in the workplace of the AU are aircraft. Noise in ground conditions is generated during the operation of power plants and auxiliary equipment (compressors, air conditioners, etc.) necessary for preparing the aircraft for flight. The noise inside the aircraft during the flight is due to the operation of engines, air conditioners and a turbulent boundary layer of air around the airframe. The analysis allows us to distinguish the following features of AS: broadband noise with the presence of several peaks; high intensity (sound pressure levels at all octave frequencies exceed $100 \mathrm{~dB}$ ); the presence of an infrasound component; the noise is unstable (periods of "active" noise load alternate with pauses); simultaneously the presence of several sources of noise in the workplace.
It has been established that in the workplaces the AS significantly exceeds the maximum permissible level in the sound and infrasound ranges. The presence in the AS spectrum of highintensity IP (total USD 101-111 dB) and acoustic vibrations of the sound range (USD 116-127 dBA) is of scientific interest, since such information is isolated in the scientific literature. The classical description of the development of pathological changes in the body when exposed to noise is well laid out practically in the manuals on otorhinolaryngology. The clinical picture of noise pathology is based on the effect of high-frequency noise. The duration of the development of a hearing organ disease until the onset of clinical symptoms of occupational HCT (PNST) is at least 5 years. The likelihood of developing hearing impairment depends on the level of noise and the length of experience with noise. Aural and extraaural effects of noise on the body are distinguished, which develop in parallel. Features of the clinical manifestations of PNST include: a slow and progressive course of the process, bilateral symmetrical decrease in tonal hearing through air and bone conduction, increased hearing thresholds in the high-frequency region without bone-air dissociation.

PNST when exposed to aircraft noise. The first clinical symptoms of it appear 2 years after the action of AS, that is, faster than when exposed to high-frequency noise. The peculiarity of the course of the disease is characterized by the fact that vegetativevascular disorders often precede hearing impairment. The nature of complaints under ASh practically does not differ from complaints in the classical form of PNST. The otoscopic picture with 
the development of PSNT from AS does not give specific clinical manifestations. The method of tonal audiometry in AS revealed the features of changes in hearing thresholds: the absence or slight severity of the noise tooth, the descending type of audiogram, the absence of a bone-air gap. Clinical signs of hearing impairment according to tonal audiometry in speakers appear two years later in the form of increased thresholds for the perception of sound tones in the region of 12-16 kHz. The likelihood of developing PNST from $\mathrm{AS}$ is higher than under the influence of high-frequency noise with an experience of more than 15 years. With a work duration of 5-10 years, in both cases it complies with the ISO standard estimates.

The clinical picture of extraaural effects in PNST from AS is characterized by the following syndromes and diseases: mixed vestibulopathy, vegetovascular dystonia, respiratory and cardiovascular diseases, that is, a combination of diseases characteristic of both noise and FM. Vascular and autonomic disorders develop in parallel with hearing impairment, and in some cases they can precede the development of HCT. Based on the foregoing, it can be argued that the nature of hearing damage, as well as the syndromes caused by the extra-aural effect of the combined effect of noise and FM, have a number of features that allow distinguishing PNST due to the combined effect of noise and FM in a separate form of HCT.

The nature of PNST under the action of ASh has the following features:

1) The presence of sound levels in the workplace above the remote control.

2) The presence of an infrasound component, the ultrasound of which exceed the remote control.

3) Professional work experience with ASh for at least two years.

4) A gradual increase in the degree of hearing loss observed during the follow-up according to medical records.

5) A downward type of audiogram with an increase in thresholds of sound perception over the entire range of frequencies under study, both in the low-frequency (125-500 $\mathrm{Hz}$ ) to $-20 \mathrm{~dB}$, and, to a greater extent, in the high-frequency range $(4000-8000 \mathrm{~Hz})$ from $30 \mathrm{~dB}$ to $40 \mathrm{~dB}$.

6) Bilateral damage to the organ of hearing.
7) Lack of bone-air dissociation.

8) The absence of other causes of hearing loss.

9) The presence of extraaural clinical manifestations in combination with HCT.

It is advisable to take these features into account when solving clinical and expert issues regarding the connection between the NST and the aviation profession. An analysis of the literature shows that the above differences between the NST formed in the Air Force AS are characteristic of specialists in a number of other industries. One of the modern features of industrial noise is an increase in the spectrum of low-frequency and infrasound components. The results of acoustic measurements show that if the noise levels are about 90-100 dBA, then we can expect the presence of IZ with an ultrasound of 100-107 dB. Sources of production noise with an infrasound component with an ultrasonic ultrasound component above $100 \mathrm{~dB}$ are all types of transport (air, rail, sea, river and automobile) and many types of industry (aviation, construction, metallurgy, mining, gas and oil production, etc.).

\section{Conclusion}

1. AS is a broadband high-intensity noise with the presence of an infrasonic component, which allows us to talk about the combined effect of noise and IZ. AS creates a risk of developing HCT.

2. The combined effect of noise and IZ leads to the development of a special form of NST, which differs from NST under the action of industrial noise, in the spectrum of which medium and high-frequency sound vibrations predominate.

3. At the Air Force AS subject to aviation noise, tonal audiometry is a mandatory measure for hiring and for dynamic observation. Identification of the initial signs of hearing impairment in them requires a clinical examination, timely initiation of treatment and an expert assessment of professional suitability.

\section{Acknowledgement}

None.

\section{Conflict of Interest}

No conflict of interest. 